\title{
Au sujet de la définition et de la réglementation des eaux minérales naturelles
}

\author{
J. Canellas \\ Université de Bordeaux 2
}

\section{Q DÉFINITION}

Si on compare les définitions de l'eau minérale qui ont été données par le passé, on constate que ces énoncés diffèrent entre autres par les nuances apportées à l'appréciation de leurs propriétés thérapeutiques. Il est ainsi possible d'envisager successivement quelques-unes de ces définitions en partant des plus formelles pour arriver aux plus nuancées.

L'affirmation des propriétés thérapeutiques se retrouve dans les définitions données par A. MORETTE et par M. LAMARCHE :

"Les eaux minérales naturelles et les gaz thermaux sont des eaux et des gaz issus de formations géologiques, reconnues par l'Académie de Médecine, douées de propriétés thérapeutiques et dont l'administration ou la livraison au public sous quelque forme que ce soit a été autorisée par arrêté ministériel »(A. MORETTE, Précis d'Hydrologie, 1964).

*On définit l'eau minérale comme étant une eau de source naturelle douée de propriétés thérapeutiques et cela quels que soient ses caractères physiques ou son degré de minéralisation» (M. LAMARCHE, Hydrologie et Climatologie médicale, 1977).

Une nuance est apportée par P. Delore (Précis d'Hydrologie et de Climatologie, 1951) « L'eau minérale est une eau de source naturelle dont l'usage est susceptible de provoquer un effet thérapeutique. "
Il faut également retenir la formule proposée par B. NINARD : "L'eau minérale est une eau naturelle d'intérêt médical » (Journal Français d'Hydrologie, $\mathrm{n}^{\circ} 37$, 1982).

Le Grand Larousse Encyclopédique (1961) apporte une nuance supplémentaire : «On nomme ainsi les eaux naturelles que leur composition physique et chimique rend susceptible de provoquer dans l'organisme humain des modifications qui peuvent être utilisées favorablement. "

"Modifications favorables", c'est l'expression que le législateur a retenu dans la définition figurant dans le décret du 6 juin 1989 relatif aux eaux minérales et aux eaux potables préemballées et que l'on doit considérer aujourd'hui comme définition officielle :

"Une eau minérale naturelle est une eau possédant un ensemble de caractéristiques qui sont de nature à lui apporter ses propriétés favorables à la santé.

Elle se distingue des autres eaux destinées à la consommation humaine:

- par sa nature caractérisée par sa teneur en minéraux. oligoéléments ou autres constituants et par certains effets ; - par sa pureté originelle.

l'une et l'autre caractéristiques ayant été conservées intactes en raison de l'origine souterraine de cette eau qui a été tenue à l'abri de tout risque de pollution.

Elle provient d'une nappe ou d'un gisement souterrain exploité à partir d'une ou plusieurs émergences naturelles ou forées.

\section{On the definition and regulation of natural mineral waters}

In the past, mineral water was defined by means of various formulae, while today the definition given in article 2 of the Decree of 6 June 1989 is used. This law sets out the main characteristics of mineral water, for example its health-giving properties, constituent concentrations, absence of any pollution risk, and the stability of its properties.

In France, the " licence to exploit from the spring " which, in fact, acknowledges the name « natural mineral water is granted by the Minister of Health following a lengthy procedure. Additional licences are necessary for the water to be used in a spa establishment or a bottling plant. In both cases, there will be surveillance by a laboratory approved by the Ministry of Health. 
Elle témoigne, dans le cadre des fluctuations naturelles connues, d'une stabilité de ses caractéristiques essentielles, notamment de composition et de température à l'émergence, qui n'est pas affectée par le débit de l'eau prélevée. »

On conçoit que ce texte ne satisfasse pas certains thermalistes attachés aux définitions mettant l'accent sur l'activité thérapeutique. Il est vrai que l'énoncé du 6 juin 1989 figure dans un texte consacré en partie aux eaux minérales embouteillées dont certaines répondent également aux paramètres définissant les eaux de consommation humaine.

Quoi qu'il en soit, l'expression consacrée pour désigner le produit demeure « eau minérale naturelle».

On pourrait penser qu'il s'agit là d'un pléonasme : en réalité, il est apparu nécessaire de rappeler que seules les eaux naturelles peuvent maintenant être qualifiées de «minérales ". En effet, le décret du 12 janvier 1922 qui réglementait les « eaux minérales artificielles " a été abrogé par le décret du 6 juin 1989 dont l'article 23 précise : « Le décret du 12 janvier 1922 modifié relatif au commerce des eaux naturelles et artificielles et des eaux de boisson est abrogé. „

\section{II 口 RÉGLEMENTATION}

\subsection{Reconnaissance d'une eau minérale naturelle}

Un certain nombre d'étapes vont séparer la découverte de l'eau de sa reconnaissance comme eau minérale naturelle.

Depuis la Loi sur l'eau (3 janvier 1992), la première démarche va concerner le forage. En effet, depuis le décret du 29 mars 1993 relatif aux procédures d'autorisation et de déclaration, un ouvrage permettant un prélèvement supérieur à $80 \mathrm{~m}^{3} / \mathrm{h}$ est soumis à autorisation alors qu'entre 8 et $80 \mathrm{~m}^{3} / \mathrm{h}$, l'ouvrage est soumis à déclaration.

Le forage étant productif, le propriétaire a intérêt à s'assurer des qualités microbiologiques et de la constance de l'eau obtenue, par exemple, par un suivi microbiologique et chimique sur une année avec examens mensuels, avant d'engager la procédure de reconnaissance.

Ces garanties étant acquises, la demande d'autorisation d'exploitation de l'eau en tant qu'eau minérale naturelle est déposée à la Préfecture du Département. Le Préfet transmet le dossier à la Direction Régionale de l'Industrie de la Recherche et de l'Environnement dont le rôle est essentiellement de s'assurer de la conformité du captage et de procéder à quelques déterminations de base (débit, température, conductivité,....). Sont également saisis par le Préfet la Direction Départementale de l'Action Sanitaire et Sociale chargée plus spécialement de l'aspect hygiène et le Laboratoire agréé pour la surveillance des eaux minérales qui effectuera deux analyses, l'une en fin de période sèche, l'autre en fin de période humide. Le Laboratoire agréé demandera également à l'Office de Protection contre les Rayonnements Ionisants de faire une analyse de radioactivité. Enfin le Médecin Inspecteur Départemental établira un rapport qui sera soumis au Conseil Départemental d'Hygiène.

L'enquête départementale se terminera à ce stade et si les résultats sont satisfaisants, le Préfet transmettra le dossier avec ses observations au Ministre chargé de la Santé.

Au sein du Ministère interviendra la Direction Générale de la Santé par l'intermédiaire de la Section Thermalisme du Bureau de l'Eau (Sous-direction de la Veille Sanitaire) et au sein de l'Agence du Médicament, l'Unité fonctionnelle du thermalisme qui effectuera deux nouvelles analyses à six mois d'intervalles (analyses qui figureront dans le rapport soumis à l'Académie de Médecine et constitueront les analyses officielles). Ces nouvelles déterminations ont pour but de s'assurer de l'absence de variation des qualités microbiologiques et des caractéristiques physico-chimiques et chimiques. L'ensemble du dossier est transmis pour avis à l'Académie de Médecine où il est examiné par la Commission du thermo-climatisme dont le Président présentera un rapport complet en séance plénière de l'Académie. Enfin, en possession de l'avis de l'Académie de Médecine, le Ministre prendra l'arrêté « d'autorisation d'exploitation de l'eau en tant qu'eau minérale naturelle. »

Dans les meilleures conditions, les diverses démarches énumérées se déroulent sur environ trois années.

\subsection{Quelques remarques}

Il apparaît souhaitable que cette procédure qui comprend déjà une étude de la radioactivité, soit complétée par une étude de l'évolution de la composition de l'eau au contact de l'atmosphère et par des examens biologiques et pharmacodynamiques. En effet, un dossier complété par quelques données pharmacologiques simples faciliterait la prise de décision des membres de l'Académie de Médecine, surtout depuis que le raisonnement par analogie (à compositions chimiques voisines: propriétés analogues) est discuté et rejeté (peut-être de façon trop absolue). S'il n'est pas possible de calquer cette procédure sur celle qui conduit à la mise sur le marché d'une spécialité pharmaceutique, il est possible d'effectuer parallèlement aux diverses opérations décrites précédemment quelques essais pharmacologiques.

En effet, à partir du moment où les analyses du Laboratoire agréé apportent des garanties de qualité, on peut envisager les essais suivants :

— Essais de type «toxicologie» :

Essai de tolérance par diverses voies : voie buccale, voie pulmonaire (aérosol),...

- Essais de pharmacodynamie :

Organes isolés : action propre, interaction vis-à-vis de divers médiateurs,...

Animal entier : transit intestinal, diurèse, action vis-à-vis du bronchospasme expérimental, métabolisme des lipides,,..

L'ensemble des données fournies par ces différentes études permettra d'établir un projet d'étude clinique correspondant à la phase I et au moins à la première partie de la phase II. L'étude clinique pourra être réalisée après avis d'un Comité Consultatif de Protection des Personnes dans la Recherche Biomédicale. Les résultats obtenus pourront être communiqués aux membres de l'Académie de Médecine. Par contre, il serait logique que la phase III qui permettra de préciser les indications et l'orientation de la Sécurité Sociale ne soit réalisée qu'après l'obtention de l'autorisation d'exploitation.

L'eau étudiée étant reconnue eau minérale naturelle, l'exploitation peut se faire soit à des fins thérapeutiques (cure thermale), soit à des fins d'embouteillage (eaux conditionnées dites préemballées). 


\subsection{Le thermalisme et l'établissement thermal}

Le thermalisme est l'utilisation à des fins thérapeutiques des produits naturels que sont les eaux minérales ou leurs dérivés, dans un établissement spécialisé (Thermes), situé dans un site privilégié, sous surveillance médicale.

Les pratiques thermales sont mises en jeu dans l'Etablissement thermal qui peut être à son tour défini de la façon suivante :

"Sont considérés comme établissements thermaux, les établissements qui utilisent sur place ou par adduction directe, pour le traitement interne ou externe des malades, l'eau d'une ou plusieurs sources minérales régulièrement autorisées ou ses dérivés : boues ou gaz."

S'il n'est pas demandé de remboursement par la Sécurité Sociale, un établissement thermal peut fonctionner sans autre autorisation au titre du Ministère de la Santé que celle donnée à l'eau minérale naturelle. Cette éventualité ne se pose pratiquement pas et pour permettre le remboursement d'une partie des frais de cure par la Sécurité Sociale, il est nécessaire de remplir un certain nombre de conditions.

Quoi qu'il en soit les règles de fonctionnement des établissements thermaux sont définies par un décret, elles seront prochainement complétées dans un Guide de Bonnes Pratiques.

La suite de la procédure permettant la prise en charge par la Sécurité Sociale comprend:

1. La reconnaissance des orientations thérapeutiques par inscription à la Nomenclature Générale des Actes Professionnels. La demande est instruite par la Direction Générale de la Santé et la Direction de la Sécurité Sociale. Il y a consultation du Haut Comité du Thermalisme et du Climatisme et de la Caisse Nationale d'Assurance Maladie des Travailleurs Salariés.

La définition des pratiques thermales sera effectuée avec le concours de la Commission Technique du Thermalisme.

2. L'agrément de l'établissement thermal. L'agrément est accordé par la Commission Régionale d'Agrément (DRASS) après enquête du Médecin Inspecteur Départemental.

3. La tarification. Le forfait thermal de base (traitement type) et les suppléments (pratiques thermales complémentaires) seront fixés par le Préfet après avis de la Direction Générale de la Concurrence, de la Consommation et de la Répression des Fraudes, de la Caisse Nationale d'Assurance Maladie, de la Commission Technique du Thermalisme.

\subsection{Surveillance}

La surveillance est assurée par les laboratoires agréés par le Ministère deux ou trois fois au cours de la saison thermale. Elle porte principalement sur la qualité sanitaire pour laquelle les exigences sont plus importantes que pour les eaux de consommation. Actuellement de nombreuses stations mettent en place un laboratoire destiné à exercer une autosurveillance.

\section{LES EAUX PRÉEMBALLÉES}

Les eaux préemballées font l'objet des décrets du 11 décembre 1964 et du 6 juin 1989. Ce dernier précise être : " applicable lorsqu'elles sont préemballées aux eaux minérales naturelles, aux eaux de sources et aux eaux rendues potables par traitements, à l'exception de celles qui sont des médicaments et de celles utilisées à la source dans les établissements de soin et de cure. ”

Le tableau situe les eaux préemballées parmi les différents types d'eau.

\subsection{Eaux minérales naturelles}

L'eau doit avoir bénéficié d'une autorisation d'exploitation en tant qu'eau minérale naturelle et d'une autorisation ministérielle d'embouteillage. La possibilité d'un embouteillage en matériau plastique fait l'objet d'une autorisation particulière. Sont autorisés (décret du 28 mars 1957 modifié par le décret du 6 juin 1989) les traitements de séparation des composés instables, notamment du fer ou du manganèse, par décantation ou filtration. C'est ainsi que diverses techniques sont mises en jeu pour permettre en particulier l'embouteillage d'eaux bicarbonatées riches en fer.

La surveillance est assurée tous les deux mois par un laboratoire agréé pour la surveillance des eaux minérales et en général de façon quasi continue par le laboratoire réalisant l'auto-surveillance.

Parmi les dix catégories d'eaux préemballées, six intéressent les eaux minérales, à savoir :

Eau minérale naturelle non gazeuse ;

Eau minérale naturellement gazeuse ;

Eau minérale renforcée au gaz de la source :

Eau minérale avec adjonction de gaz carbonique ;

Eau minérale naturelle totalement dégazéifiée :

Eau minérale naturelle partiellement dégazéifiée.

Les eaux minérales naturelles préemballées peuvent bénéficier d'une appellation rappelant leur composition chimique, exemple :

Eau sulfatée si $\mathrm{SO}_{4}^{--}>200 \mathrm{mg} / \mathrm{l}$;

Eau calcique $\mathrm{si} \mathrm{Ca}^{++}>150 \mathrm{mg} / \mathrm{l}$;

Eau sodique si $\mathrm{Na}^{+}>200 \mathrm{mg} / \mathrm{l}$;

Eau bicarbonatée si $\mathrm{HCO}_{3}^{-}>600 \mathrm{mg} / \mathrm{l}$;

Eau magnésienne si $\mathrm{Mg}^{++}>50 \mathrm{mg} / \mathrm{l}$;

Eau chlorurée $\mathrm{si} \mathrm{Cl}^{-}>200 \mathrm{mg} / \mathrm{l}$;

Eau fluorée ou fluorurée si $\mathrm{F}^{-}>1 \mathrm{mg} / \mathrm{l}$;

Eau convenant à un régime pauvre en sodium si $\mathrm{Na}^{+}<20 \mathrm{mg} / \mathrm{l}$;

$\cdots$

\subsection{Eau de source}

Ces eaux sont d'origine souterraine et naturellement potables microbiologiquement et chimiquement. Elles ne doivent subir aucun traitement (sauf la séparation d'éléments instables ou de matières en suspension par décantation ou filtration (sans modification de la composition chimique) et l'incorporation de gaz carbonique). Les traitements proposés ne doivent en aucun cas avoir pour but ou effet de modifier les caractéristiques microbiologiques (désinfec- 
Tableau 1. - Les différents types d'eau et leurs parcours.

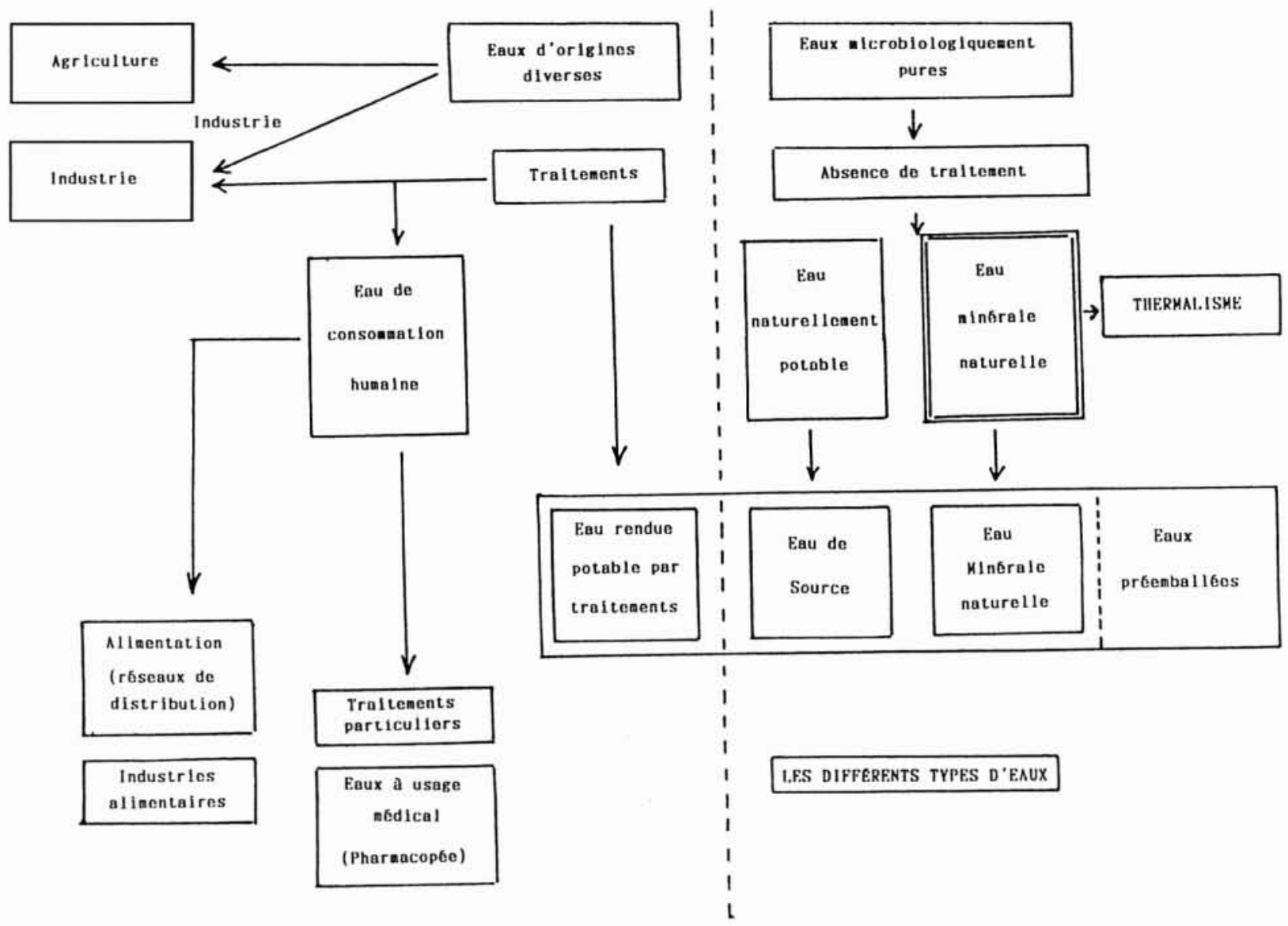

tion dissimulée). L'autorisation d'embouteillage est accordée localement par le Préfet du département.

La surveillance est assurée par le laboratoire départemental.

\subsection{Eau rendue potable par traitement}

Ce type d'eau d'intérêt limité était désigné autrefois par l'appellation " Eau de table ".

L'indication des traitements subis par l'eau est obligatoire. L'adjonction de gaz carbonique est autorisée, par contre, les vocables minéral et source sont interdits et il ne peut être fait mention de propriétés favorables à la santé ou d'un élément particulier de la composition de l'eau.

La surveillance est assurée par le laboratoire départemental.

\subsection{Quelques considérations sur les eaux préemballées}

La plupart des eaux minérales embouteillées se différencient des eaux minérales «telles qu'à l'émergence ». par la disparition de certains éléments par suite des traitements autorisés pour l'embouteillage (déferrisation), de l'oxygénation qui provoque la disparition des éléments oxydables, parmi lesquels certains oligoéléments, des phénomènes d'adsorption sur les parois des récipients (cas du verre), de la disparition du radon,... Ces altérations présentent plus ou moins d'importance selon le type d'eau considéré. Les modifications peuvent être telles que, dans les conditions classiques d'embouteillage, l'eau perdra ses caractéristiques essentielles (eaux sulfurées). Cependant, il existe des procédés de stabilisation permettant de conserver en solution le fer et autres éléments oxydables. Ces conditionnements sont vendus en pharmacie (ex.: Hydroxydase).

Les eaux minérales se différencient des eaux de source car les normes de potabilité chimique des eaux de consommation humaine appliquées aux eaux de source ne peuvent l'être aux eaux minérales qui se caractérisent par leur minéralisation souvent plus élevée, leur teneur en sulfate, en bicarbonate, en arsenic, en fluor...

La différence avec les eaux rendues potables par traitement est encore plus grande puisque ces dernières sont, comme les eaux de distribution, des eaux ayant subi différentes opérations : floculation, filtration, désinfection...

\section{CONCLUSION}

L'autorisation d'exploitation qui reconnaît le caractère «eau minérale naturelle» n'est accordée, du moins actuellement, qu'après une longue procédure justifiant des "propriétés favorables à la santé. " 
Il est toutefois possible de considérer que si cela peut se révéler très suffisant dans le cas de certaines eaux embouteillées ou de l'utilisation de l'eau dans diverses techniques de détente où l' " hydrothérapie " est associée à diverses activités sportives, on conçoit qu'il soit envisageable de demander davantage dans le cas de la « crénothérapie » c'est-à-dire de la cure thermale.

\section{ANNEXE}

\section{Les eaux gazeuses et/ou gazéifiées}

Compte tenu de leur importance dans le marché des eaux embouteillées, de leur possibilité d'appartenir aux diffé- rentes catégories d'eaux préemballées et de leur utilisation en dehors de toute considération thérapeutique, il semble utile de faire une place aux eaux gazeuses et/ou gazéifiées en précisant la signification des deux appellations.

C'est ainsi que l'on trouvera :

Eau minérale naturelle naturellement gazeuse (ou eau minérale naturelle gazeuse) ;

Eau minérale naturelle renforcée au gaz de la source (gazeuse) ;

Eau minérale naturelle avec adjonction de gaz carbonique (gazéifiée) ;

Eau de source avec adjonction de gaz carbonique (gazéifiée) ;

Eau rendue potable par traitement et avec adjonction de gaz carbonique (gazéifiée). 